\title{
Comparison between different statistical models for the prediction of direct genetic component on embryo establishment and survival in Italian Brown Swiss dairy cattle
}

\author{
F. Tiezzi ${ }^{\mathrm{b}}$, C. Maltecca ${ }^{\mathrm{b}}$, A. Cecchinato ${ }^{\mathrm{a}, *}, \mathrm{G}$. Bittante ${ }^{\mathrm{a}}$ \\ a Department of Agronomy, Food, Natural resources, Animals and Environment, University of Padova, Viale dell'Università 16, 35020 Legnaro, PD, Italy \\ ${ }^{\mathrm{b}}$ Department of Animal Science, North Carolina State University, Raleigh 27695, NC, USA
}

\section{A R T I C L E I N F O}

\section{Article history:}

Received 4 November 2014

Received in revised form

9 April 2015

Accepted 30 June 2015

\section{Keywords:}

Bull fertility

Heritability

Model comparison

Threshold model

\begin{abstract}
A B S T R A C T
The aims of this study were to infer variance components and heritability for the direct component on embryo establishment and survival related traits and to compare different statistical models in terms of goodness-of-fit and predictive ability. Embryo establishment and survival (EES) was defined as the outcome of an AI event, its direct effect was represented as the effect of the service sire from which semen was taken. Indicators of EES were calving per service (CS) and non-return at $56 \mathrm{~d}$ after service (NR56). Insemination records from the Italian Brown Swiss population reared in the Alps were used. Data included 124,206 inseminations performed by 86 technicians on 28,873 cows in 1400 herds. Services were recorded from 1999 to 2008. Linear-sire, linear-animal, threshold-sire, and threshold-animal models were used to estimate (co)variance components for CS and NR56. Four levels of complexity within each model were tested, so that 16 different models were compared for each of the two fertility traits. Comparison was assessed on the basis of the goodness-of-fit and predictive ability. Paternal halfsibs groups were created as average outcome of the inseminations from a given service sire. Goodnessof-fit was evaluated by regressing the service sire estimated breeding value from each model to paternal half-sibs average CS or NR56. Predictive ability was assessed through sums of chi-squared and percentage of wrong predictions. Predictors were the respective service sire's estimated breeding values constructed on a reduced (independent) training dataset, including years from 1999 to 2005, and predictands were the paternal half-sibs means for every bull in the remaining years (2006-2008). Prediction of EES was considered differently according to whether service sires had observations in the training dataset (prediction of proven bulls) or they had not (prediction of young bulls). Estimates of heritability ranged from 0.011 to 0.119 for CS, and from 0.005 to 0.054 for NR56. In general, threshold models explained a larger proportion of additive genetic variance than linear models, and animal models yielded higher heritabilities than sire models. Calving per service was much more predictable than NR56, but no significant differences were found among models. Although heritabilities were low, the prediction of future EES of a paternal half-sib group is feasible.
\end{abstract}

(c) 2015 Published by Elsevier B.V.

\section{Introduction}

In investigating the loss of reproductive fitness in dairy cattle, female fertility has received considerable attention than its male counterpart. Low reproductive performances have been often ascribed to cow metabolism, as energy redirected to the mammary gland would hamper correct reproductive function (Veerkamp et al., 2003), and the antagonistic effect of pleiotropic genes affecting milk yield and fertility would play a relevant role (Royal

\footnotetext{
* Corresponding author. Fax: +39049827 2633.

E-mail address: alessio.cecchinato@unipd.it (A. Cecchinato).
}

et al., 2000; Lucy, 2001; Pryce et al., 2004). Nonetheless, there is evidence of the impact of male fertility on the efficiency of the dairy industry (Nadarajah et al., 1988; Clay and McDaniel, 2001; Blaschek et al., 2011).

The establishment and survivability of the embryo (otherwise called embryo establishment and survival, EES) has been recognized as a component of the whole reproductive performance (Bamber et al., 2009; VanRaden and Miller, 2006), but its impact has been seldom investigated. The EES meant as a trait of the potential calf (Azzam et al. 1988) and the phenotype is determined as the calf survival from spermatozoon to birth (or absence of rebreeding within $56 \mathrm{~d}$ from insemination).

The EES is composed of different features, from both male and 
female fertility (Ayalon, 1978). First, the fertilizing ability of the sperm cells plays a role together with the potentiality of the oocytes to be fertilized. Secondly, if conception successfully occurs, the uterine environment determines if the embryo will proceed to the cellular differentiation and the fetus will born giving a viable calf. It is intuitive that there will be a component of EES coming from the sire through the genes transmitted, i.e. a direct additive genetic effect. Also, the quality of the sperm cells affects conception success but is not transmitted to the embryo, therefore considered as an environmental effect of the service sire (Jansen, 1986). On the female counterpart, the dam is contributing to the viability of the embryo with the genes that are transmitted (maternal additive genetic effect) as well with providing a fertilizable oocyte and a uterine environment suitable for fetus growth (maternal environmental effect). Besides, other abiotic factors (climatic variation) together with management (herd level of fertility, insemination technician skills) are to be considered among the sources of variation for embryo establishment and survival.

Fertility success traits, such as non-return at $56 \mathrm{~d}$ after service (NR56) and calving per service (CS), can be used as measures of embryo survival, and can be easily derived from insemination records. While NR56 simply indicates the non-mating of the cow within $56 \mathrm{~d}$ after insemination, CS assigns success to inseminations followed by a registered calving. As the two traits are computed differently and none of them can give an incontrovertible measure of reproductive efficiency assessing embryo losses at different pregnancy timepoints, their simultaneous consideration in a study can improve fertility (and embryo survival) assessment (Sun and Su, 2010).

Estimates of heritability for EES as meant it in the present study are scarce and inconsistent. VanRaden and Miller (2006) found negligible direct and maternal heritabilities for embryo and fetal loss in US Holsteins defined as non-return rate at $70 \mathrm{~d}$ using field data analyzed with a linear model. On the other hand, Bamber et al. (2009) used data from previous reproductive management trials to estimate heritability for pregnancy loss. The higher quality of the data coupled with the used of the threshold model yielded substantially higher heritabilities ( 0.489 for the direct component and 0.166 for the maternal component). In similar studies, LópezGatius et al. (2002) and Starbuck et al. (2004) found that pregnancy retention was dependent on the service bull used, but no additive genetic effect was estimated. When EES was assumed as a trait of the calf it has been referred to as bull fertility (Azzam et al., 1988 ) but the direct additive genetic component were found to be extremely low. Andersen-Ranberg et al. (2003) used a linear model to analyze non-return rate after $50 \mathrm{~d}$ on Norwegian heifers and first-parity cows, but heritabilities were always below $2 \%$. Hyppänen and Juga (1998) also used a linear model to analyze nonreturn after $60 \mathrm{~d}$ in Finnish dairy cattle, and found null estimates of heritability. Kuhn and Hutchinson (2008) used both linear and threshold models to estimate heritability of conception rate from filed data in US Holstein cattle, and both models gave almost null value of heritability. It could be inferred that some variance can be attributed to direct and maternal genetic components for embryo survival, however the possibility of estimating non-null values of heritability depends on the quality of the data. Field data can hamper the estimation of these small genetic effects.

Selection candidates' EBV for EES may also play an important role in prediction of future performance and selection of best candidates for next generation. The most used and reliable tool for the assessment of model predictive ability is cross-validation, which has already been exploited in dairy cattle (Caraviello et al., 2004; González-Recio et al., 2005; Vazquez et al., 2012) and other species (Matos et al., 1997a; Cecchinato et al., 2010).

Genes transmitted to the embryo from the service sire, and affecting its establishment, are recognizable as direct additive genetic effect on EES. Predicting performance of a service sire for offspring embryo establishment and survival might help in improving overall fertility in artificially inseminated dairy cattle. Therefore, the purpose of this study was to investigate the impact of the direct additive genetic effect on EES of dairy cattle comparing different traits (CS and NR56), pedigree structures (sire and animal models associated to 'sire-maternal grandsire' and 'siredam' pedigrees, respectively), distributional assumptions (linear and threshold models), and model specifications for random effects. Different statistical models were compared in terms of goodness-of-fit and predictive ability of service sire future records.

\section{Materials and methods}

\subsection{Data}

Insemination records on Brown Swiss cows were obtained from the Breeders Association of Bolzano-Bozen province (northeast Italy). The same original dataset was used for previous studies on male fertility by Tiezzi et al. (2013). More than 200,000 single inseminations performed on Brown Swiss cows and heifers between 1999 and 2008 were available. Inseminations were validated as successful for CS when giving an acceptable pregnancy length of $288 \pm 15$ d (mean value for Brown Swiss from Norman et al., 2009). If two inseminations fell within this range of pregnancy length, the latter was considered successful. If pregnancy length of a heifer or a cow was lower than $273 \mathrm{~d}$, the animal was not validated as pregnant. Furthermore, cows having inseminations in a given lactation were required to have recorded inseminations on the previous parities, such that cows that showed gestation length shorter than $273 \mathrm{~d}$ were not validated as pregnant for that lactation and were eliminated for the subsequent lactations. Non-return at $56 \mathrm{~d}$ was also calculated for every service, validated as non-returned $(\mathrm{NR} 56=1)$ if no inseminations were performed within $56 \mathrm{~d}$, regardless of whether the non-return animal conceived or not. Approximately 30\% of inseminations on Brown Swiss cows reared in the Alps are conducted using semen of beef bulls to produce crossbred calves for veal and beef production (Dal Zotto et al., 2009; Penasa et al., 2009). However, most of the heifers and the majority of the cows are mated to Brown Swiss bulls. Thus, from the whole set we retained inseminations from AI Brown Swiss bulls as service sire which represent approximately $70 \%$ of total inseminations. Fertility traits (CS and NR56) were computed before the extraction of Brown Swiss bulls, because completeness of data is needed. Service sires were required to have at least 100 inseminations, with herds and technicians requiring at least 20 inseminations. Furthermore cows with only 1 service, and sires of cows with less than 20 services on the respective daughters were not considered. Lenient editing criteria on cows and sires of cows were adopted, since female fertility was a nuisance variable in the present study. Service sires, herds, technicians, and sires of cow with an average CS and NR56 outside the $0.10-0.90$ range were omitted from the analysis. Although interactions between effects were not fitted (e.g., technician by service sire), restrictions were imposed so that technicians were required to operate in more than one farm, and in each farm at least 2 technicians were required to be recorded. This was adopted for service sires, technicians, herds, and sires of cows (e.g., each sire of cow was imposed to have daughters in at least 2 farms). After editing, 124,206 single inseminations performed by 86 technicians on 28,873 cows in 1400 herds were available for analysis in the main dataset (DATA $\mathrm{DOT}_{\mathrm{TO}}$ ). 


\subsection{2 Statistical analyzes}

Linear-sire, threshold-sire, linear-animal, and threshold animal models were applied to CS and NR56.

\subsubsection{Sire and animal models}

In constructing sire and animal models, different pedigree structures were investigated. For sire models, a single sire-maternal grandsire pedigree was considered, tracing back generations starting with service sires and sires of cows as animals with phenotype. For animal models, the embryo (or potential calf) resulting from every 'service sire-cow' mating was considered as the phenotype and generations were traced back starting from the embryos.

\subsubsection{Linear and threshold models}

Although the assumptions of the linear model are violated for the traits analyzed, linear mixed models are still a popular choice in the analysis of binary traits as compared to threshold models (Gianola, 1982) as they often perform similarly and have reduced computational complexity. In our analysis, a single threshold was considered and an underlying continuous variable called liability was generated from data, according to the following assumption:

$y_{i}=\left\{\begin{array}{l}0 \text { if } \lambda \leq \tau \\ 1 \text { if } \lambda>\tau\end{array}\right.$

where $y_{i}$ is the $i$ th observation (0/1), $\lambda$ is the liability, and $\tau$ is the given threshold. In accordance to the formula, every observation $y_{i}$ takes value 1 (success for CS or NR56) if the liability is greater than $\tau$, or 0 (failure in CS or NR56) otherwise. The liability is assumed to follow a normal distribution with mean $\mu$ and variance $\sigma^{2} \lambda$. Since the mean and variance of liability are unobserved, the parameters of the model are not identifiable, and thus $\sigma^{2}{ }_{e}$ was fixed to ' 1 ' and $\tau$ to ' 0 '. Therefore, no sampling of the threshold value is needed.

\subsubsection{Model specifications}

Within the comparisons reported above, four different model structures increasing in complexity were considered.

Animal permanent environmental and genetic effects were added sequentially as follows:

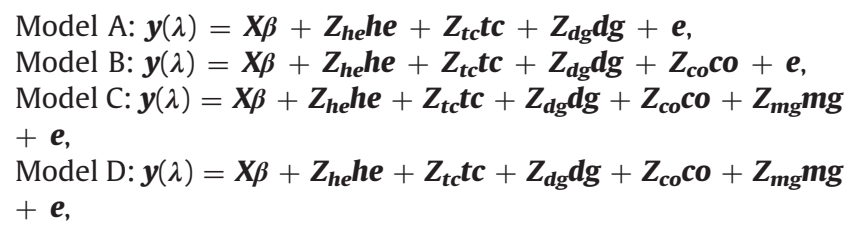

where $\boldsymbol{y}$ is the binary observation $(0 / 1)$ for linear models, $\lambda$ is the unobserved liability for threshold models, $\boldsymbol{\beta}$ is the vector of 'fixed' effects, he is the vector of herd effect, tc is the vector of technician effect, $\boldsymbol{d g}$ is the vector of direct genetic effect, $\boldsymbol{c o}$ is the vector of permanent environmental effect of the cow, $\boldsymbol{m g}$ is the vector of maternal genetic effects, ss is the vector of permanent environmental effect of the service sire, and $\boldsymbol{e}$ is the vector of residuals. $\boldsymbol{X}, \boldsymbol{Z}_{\boldsymbol{h e}}, \boldsymbol{Z}_{\boldsymbol{t c}}, \boldsymbol{Z}_{\boldsymbol{d g}}, \boldsymbol{Z}_{\boldsymbol{c o}}, \boldsymbol{Z}_{\boldsymbol{m g}}$, and $\boldsymbol{Z}_{\boldsymbol{s s}}$ are the corresponding incidence matrices of the appropriate order. Model A included direct genetic effects, accounted by a service sire effect in sire models and an embryo (or potential calf) effect in animal models. Both were assumed normally distributed with mean 0 and variance $\boldsymbol{A} \sigma_{d g}^{2}$, where $\boldsymbol{A}$ is the appropriate relationship matrix. Model $B$ was similar to model $A$ with the inclusion of permanent environmental effect of the cow in order to account for any effect of the cow on embryo establishment and survival and non-random mating of bulls to cows. In model B, the genetic and permanent environmental effects of the cow could not be disentangled. Cows were assumed unrelated among them $\left[\boldsymbol{c o} \sim N\left(0, \boldsymbol{I} \sigma^{2} \boldsymbol{c o}\right)\right]$. In model C, the maternal genetic effect represented by sires of cows in sire models and cows in animal models was added. Cows (in animal models) or sires of cows (in sire models) were assumed related among them $\left[\boldsymbol{m g} \sim N\left(0, A \sigma^{2} \boldsymbol{m g}\right)\right]$, with the appropriate relationship matrix. Here direct-maternal covariance was estimated. In model $D$, the permanent environmental effect of the service sire was added beside the direct additive genetic effect. This effect was supposed to adsorb the variation attributable to the service sire for the quality of its semen. Service sires were considered unrelated for this environmental effect $\left[\mathbf{s S} \sim N\left(0, \mathbf{I} \sigma^{2} \boldsymbol{s s}\right)\right]$.

All models included the 'fixed' effects of year-season of insemination (40 levels), parity by days in milk at insemination (age at insemination for heifers, 26 levels); status of the service sire at insemination ( 2 levels: progeny testing and proven), and the random effects of herd $\left[\boldsymbol{h e} \sim N\left(0, \mathbf{I} \sigma^{2}{ }_{h e}\right)\right]$, technician $\left[\boldsymbol{t c} \sim N\left(0, \mathbf{I} \sigma^{2} \boldsymbol{t c}\right)\right]$, and residual $\left[\mathbf{e} \sim N\left(0, \boldsymbol{I} \sigma^{2}{ }_{e}\right)\right]$.

(Co)variance components for all models were estimated in a Bayesian framework via Gibbs sampling algorithm. Flat priors were assumed for all 'fixed' effects, while for the random effects normal prior distributions were assumed. For all the computations the software TM by Legarra et al. (2008) was used. For all models 550,000 iterations were run with the first 50,000 discarded as burn-in. Thinning occurred every 50 iterations, storing 10,000 samples from every computation for inferences. Due to autocorrelations between successive samples, convergence was tested using Geweke's Z-criterion (Geweke, 1992). Monte Carlo sampling errors and the effective sample size (ESS) were computed using the time-series procedures described by Geyer (1992).

Heritability $\left(h^{2}\right)$ for sire and animal models was computed as follows:

$h^{2}($ sire $)=\frac{4 \sigma_{d g}^{2}}{\sigma_{d g}^{2}+\sigma_{s s}^{2}+\sigma_{c o}^{2}+\sigma_{m g}^{2}+\sigma_{d g-m g}^{2}+\sigma_{h e}^{2}+\sigma_{t e}^{2}+\sigma_{e}^{2}}$

$h^{2}($ animal $)=\frac{\sigma_{d g}^{2}}{\sigma_{d g}^{2}+\sigma_{s s}^{2}+\sigma_{c o}^{2}+\sigma_{m g}^{2}+\sigma_{d g-m g}^{2}+\sigma_{h e}^{2}+\sigma_{t e}^{2}+\sigma_{e}^{2}}$

where $\sigma_{d g}^{2}, \sigma_{s s,}^{2}, \sigma_{c o}^{2}, \sigma_{m g}^{2}, \sigma_{h e}^{2}, \sigma_{t e}^{2}$, and $\sigma_{e}^{2}$ are the variance components described above, and $\sigma_{d g-m g}$ is the service sire-sire of cow covariance in sire models and embryo-cow covariance in animal models. Variance and covariances reported in formulas were omitted from the denominator when not estimated (Models $\mathrm{A}-\mathrm{C}$ ). Means, and lower and upper bounds of the $95 \%$ highest posterior probability density regions for heritabilities were estimated from the Gibbs samples.

\subsection{Model comparison}

\subsubsection{Validation study}

The DATA TOT $_{\text {was }}$ partitioned into two subsets according to a year-split approach criterion. The calibration dataset $\left(\right.$ DATA $\left._{05}\right)$ started at year 1999 and was truncated at year 2005. Validation dataset $\left(\right.$ DATA $_{08}$ ) contained years from 2006 to 2008. (Co)variance components were calculated both for $\mathrm{DATA}_{\mathrm{TOT}}$ and $\mathrm{DATA}_{05}$. Sire solutions were sampled running the Gibbs sampler with fixed variance components equal to the mean value of posterior distribution of the respective (co)variances obtained previously from models A-D. In addition, an alternative predictor was calculated from model $D$ (called $D+$ ), as the sum of additive genetic and permanent environmental solutions for the service sire. DATA ${ }_{\text {TOT }}$ generated $\mathrm{EBV}_{\text {TOT }}$ and $\mathrm{DATA}_{05}$ generated $\mathrm{EBV}_{05}$.

Two different predictions were performed in order to compare models. First, average CS and NR56 in DATA ${ }_{08}\left(\mathrm{CS}_{08}\right.$ and NR56 $6_{08}$, 
respectively) for each bull was compared with the respective $E B V_{05}$ based on pedigree information (service sires excluded from DATA $_{05}$ were included in the relationship matrix). This was called 'prediction of the young bulls'. Subsequently, only service sires showing at least 100 observations in DATA 05 and 30 observations in $\mathrm{DATA}_{08}$ were considered. Their $\mathrm{EBV}_{05}$ was compared with $\mathrm{CS}_{08}$ and NR56 $6_{08}$, in order to compare the bull's breeding value from estimated first inseminations to its subsequent outcomes, calling this method 'prediction of proven bulls'.

\subsubsection{Goodness of fit}

Goodness of fit was assessed using the local weighted regression (Cleveland and Loader, 1996) between the EBV with each model (A-D and D+) and the phenotypic mean of the

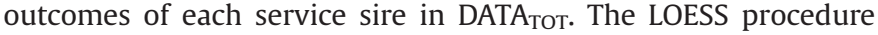
(SAS Inst. Inc., Cary, NC) was used for computing regression parameters. The procedure was set in order to choose the best smoothing parameter from each model according to the Akaike's information criterion (AIC). Models were compared in terms of mean squared error (MSE).

\subsubsection{Predictive ability}

Preditive ability was assessed for each model via sum of $\chi^{2}$ (Caraviello et al., 2004; González-Recio et al., 2005; Cecchinato et al., 2010). Binary indicators of success in DATA 08 were regressed (using logistic regression) on $\mathrm{EBV}_{05}$ obtained from each model such that the $\mathrm{EBV}_{05}$ could be converted into the service sire probability of conception for both traits. These were multiplied by the number of observations for every service sire in DATA $_{08}$ to obtain an expected number of success and failures. The value of $\chi^{2}$ was calculated for every service sire between the expected success and failures deriving from each $\mathrm{EBV}_{05}$ and the observed success and failures in DATA 08

$\chi^{2}=\left[(\text { expected success }- \text { observed success })^{2}+(\right.$ expected failures-observed failures $)^{2}$ ]

The $\chi^{2}$ values were summed across sires such that each model generated a single sum of $\chi^{2}$, which was used in model comparison. In the above equation, smaller values are preferred.

We also computed the number of wrong predictions on total predictions (WP), as difference between expected and observed successful inseminations (either for CS and NR56) divided by total number of inseminations for that service sire. The median of the service sire values for each model was reported (smaller values preferred).

\section{Results and discussion}

\subsection{Descriptive statistics}

Descriptive statistics for the data used in the study are in Table 1. The final dataset $\left(\mathrm{DATA}_{\mathrm{TOT}}\right)$ consisted of 124,206 records distributed over 40 classes of year-season of breeding, 26 classes of parity by days in milk of the cow, and 2 classes of status of the bull at service. Inseminations were performed by 86 technicians on 28,873 cows (progeny of 514 sires) reared in 1400 herds. There were 306 service sires which averaged 406 inseminations each, with a minimum of 100 and a maximum of 8255 inseminations. The pedigree file included 1292 individuals in the sire-maternal grandsire model, and 176,829 in the animal model. The overall mean for CS and NR56 was 0.48 and 0.70 , respectively. It should be noted that $22 \%$ of data were discordant, giving success for NR56 and failure for CS. Non-return at $56 \mathrm{~d}$ after service is weakly associated to CS and therefore appears as a moderate indicator of reproductive efficiency if we assume CS to be the one most closely reflecting real fertility of the cow.
Table 1

Descriptive statistics for the whole edited dataset ${ }^{\mathrm{a}}$ (DATA (DOT) $_{\text {) }}$ and calibration datase $^{\mathrm{b}}\left(\mathrm{DATA}_{05}\right)$ used to obtain service sire EBVs to be used in the cross-validation.

\begin{tabular}{lll}
\hline Item & DATA $_{\text {TOT }}$ & DATA $_{05}$ \\
\hline Number of inseminations in the dataset & 124,206 & 85,244 \\
Average calving per service (CS) & 0.48 & 0.49 \\
Average non-return at 56 d after service (NR56) & 0.70 & 0.70 \\
Percentage of discordant values (NR56=1 and CS $=0$ ) & 22 & 21 \\
$\quad$ (CSCS =CS $=0$ ) & 306 & 233 \\
Number of service sires & 100 & 100 \\
Minimum number of inseminations per service sire & 406 & 366 \\
Average number of inseminations per service sire & 0.45 & 0.46 \\
Mean of average CS per service sire ${ }^{c}$ & 0.69 & 0.69 \\
Mean of average NR56 per service sire & & 23,889 \\
Number of cows & 28,873 & 1 \\
Minimum number of inseminations per cow & 2 & 450 \\
Number of sires of cow & 514 & 1 \\
Minimum number of inseminations per sire of cow & 20 & 1398 \\
Number of herds & 20 & 4 \\
Minimum number of inseminations per herd & 86 & 83 \\
Number of technicians & 25 & 18 \\
Minimum number of inseminations per technician & & \\
\hline
\end{tabular}

a Whole edited dataset refers to years 1999-2008.

${ }^{\mathrm{b}}$ Calibration dataset refers to years 1999-2005. Minimum frequency for the random effects does not apply here.

${ }^{c}$ Average service sire outcomes are representative of the paternal half-sib groups of embryos.

The calibration dataset $\left(\mathrm{DATA}_{05}\right)$ included 85,244 records, i.e. $69 \%$ of the entire data (Table 1 ). All the 26 classes of parity by DIM of the cow and the 2 classes of the status of the bull at service were represented, whereas only 28 out of the 40 year-season classes of breeding were enclosed. For random effects, 1398 herds, 83 technicians, 450 sires of cow, and 233 service sires were considered in the analysis. Service sires averaged 366 records and 50\% of them had more than 180 observations.

\section{2. (Co)variance components and heritability}

Estimates for the (co)variance components and heritabilities for CS and NR56 (Tables 2 and 3, respectively) were the means of the posterior distributions for the respective parameters. All estimates were low, reflecting the difficulty to assess factors associated to male fertility from field data, or the low impact that the random effects included in the analysis have on field fertility.

Concerning CS, herd $\left(\sigma_{h e}^{2}\right)$ and technician $\left(\sigma_{t c}^{2}\right)$ variances remained quite stable across all models, with the former being larger than the latter, and both being much smaller than the residual variance (Table 2). Results within linear and threshold models, despite the different scale on which the solutions are expressed, showed that direct genetic variance $\left(\sigma_{d g}^{2}\right)$ was seldom higher when estimated from animal than sire models. Moreover, $\sigma_{d g}^{2}$ remained almost unchanged across sire models $\mathrm{A}-\mathrm{C}$, and decreased in model $\mathrm{D}$, whereas it gradually decreased across animal models A-D. Variances are not comparable between linear and threshold models, due to the constraint on residual variance. Regardless of the approach used, permanent environmental variance of the cow $\left(\sigma^{2}\right.$ co $)$ decreased once maternal genetic effect was included. Maternal genetic variance $\left(\sigma^{2}{ }_{m g}\right)$ was essentially constant across all models where was included. Permanent environmental variance of the service sire $\left(\sigma^{2}{ }_{s s}\right)$ was higher when estimated from threshold than linear models. The direct-maternal genetic covariance $\left(\sigma_{d g-m g}\right)$ was always negative, and the highest estimates were from threshold models, particularly from models C (Table 2). Given the low direct and maternal genetic variances, the resulting directmaternal genetic correlation ranged from -0.020 for linear sire model D to -0.563 for linear animal model C (data not shown). For NR56, posterior means of (co)variance components followed 
Table 2

Estimates $^{\mathrm{a}}$ of variance components and heritability for calving per service (CS) from different models ${ }^{\mathrm{c}}$.

\begin{tabular}{|c|c|c|c|c|c|c|c|c|c|c|}
\hline & \multicolumn{8}{|c|}{ (Co)variance components ${ }^{\mathrm{b}}$} & \multicolumn{2}{|c|}{ Heritability } \\
\hline & $\sigma_{d g}^{2}$ & $\sigma_{c o}^{2}$ & $\sigma_{m g}^{2}$ & $\sigma_{d g-m g}$ & $\sigma_{s s}^{2}$ & $\sigma_{h e}^{2}$ & $\sigma_{t c}^{2}$ & $\sigma^{2}{ }_{e}$ & $h^{2}$ & HPD95 \\
\hline \multicolumn{11}{|c|}{ Linear sire model } \\
\hline A & 0.0028 & & & & & 0.0035 & 0.0013 & 0.2410 & 0.045 & $0.034 ; 0.057$ \\
\hline B & 0.0028 & 0.0098 & & & & 0.0031 & 0.0014 & 0.2318 & 0.044 & $0.033 ; 0.057$ \\
\hline C & 0.0028 & 0.0087 & 0.0015 & -0.0004 & & 0.0030 & 0.0014 & 0.2318 & 0.045 & $0.034 ; 0.058$ \\
\hline $\mathrm{D}$ & 0.0007 & 0.0087 & 0.0015 & -0.0004 & 0.0016 & 0.0030 & 0.0014 & 0.2318 & 0.011 & $0.002 ; 0.025$ \\
\hline \multicolumn{11}{|c|}{ Linear animal model } \\
\hline A & 0.0204 & & & & & 0.0031 & 0.0014 & 0.2269 & 0.081 & $0.068 ; 0.094$ \\
\hline B & 0.0118 & 0.0068 & & & & 0.0030 & 0.0014 & 0.2266 & 0.047 & $0.038 ; 0.058$ \\
\hline C & 0.0110 & 0.0039 & 0.0101 & -0.0063 & & 0.0028 & 0.0014 & 0.2267 & 0.042 & $0.034 ; 0.051$ \\
\hline $\mathrm{D}$ & 0.0061 & 0.0042 & 0.0083 & -0.0034 & 0.0011 & 0.0028 & 0.0014 & 0.2288 & 0.024 & $0.014 ; 0.037$ \\
\hline \multicolumn{11}{|c|}{ Threshold sire model } \\
\hline A & 0.0196 & & & & & 0.0229 & 0.0090 & 1 & 0.075 & $0.057 ; 0.095$ \\
\hline B & 0.0205 & 0.0668 & & & & 0.0218 & 0.0096 & 1 & 0.073 & $0.055 ; 0.095$ \\
\hline C & 0.0210 & 0.0587 & 0.0107 & -0.0032 & & 0.0212 & 0.0097 & 1 & 0.075 & $0.057 ; 0.095$ \\
\hline $\mathrm{D}$ & 0.0052 & 0.0588 & 0.0106 & -0.0026 & 0.0119 & 0.0213 & 0.0097 & 1 & 0.018 & $0.004 ; 0.043$ \\
\hline \multicolumn{11}{|c|}{ Threshold animal model } \\
\hline A & 0.1392 & & & & & 0.0222 & 0.0100 & 1 & 0.119 & $0.102 ; 0.137$ \\
\hline B & 0.0837 & 0.0472 & & & & 0.0215 & 0.0100 & 1 & 0.072 & $0.058 ; 0.087$ \\
\hline C & 0.0775 & 0.0278 & 0.0676 & -0.0420 & & 0.0203 & 0.0101 & 1 & 0.062 & $0.050 ; 0.074$ \\
\hline $\mathrm{D}$ & 0.0401 & 0.0280 & 0.0600 & -0.0245 & 0.0093 & 0.0200 & 0.0099 & 1 & 0.034 & $0.018 ; 0.049$ \\
\hline
\end{tabular}

a Estimates are the means of the marginal posterior densities for variance components and the mean for the heritability; HPD95: lower and upper bound of the 95\% highest posterior density region.

${ }^{\mathrm{b}} \sigma_{d g}^{2}$ is the direct genetic variance; $\sigma_{c o}^{2}$ is the permanent environmental variance of the cow; $\sigma^{2}{ }_{m g}$ is the maternal genetic variance; $\sigma_{d g-m g}$ is the direct-maternal genetic covariance; $\sigma_{s s}^{2}$ is the permanent environmental variance of the service sire; $\sigma^{2}{ }_{h e}$ is the herd variance; $\sigma^{2}{ }_{t c}$ is the technician variance; and $\sigma^{2}{ }_{e}$ is the residual variance.

${ }^{c}$ All models account for the 'fixed' effects of year-season of insemination, parity by days in milk of cow at insemination (age at insemination for heifers), status of the

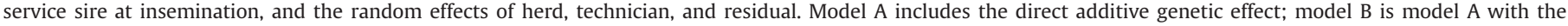

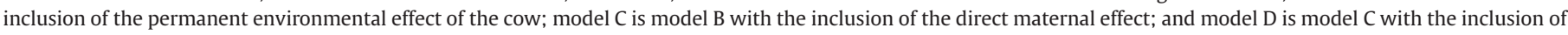
the permanent environmental effect of the service sire.

the same pattern described for CS, although they were lower (Table 3).

The posterior means of heritability for CS (0.011-0.119; Table 2) were higher than those obtained for NR56 (0.005 to 0.054; Table 3). Regarding CS, threshold gave higher heritabilities than linear models (0.018-0.119 vs. 0.011-0.081), and animal provided higher heritabilities than sire models (0.024-0.119 vs. 0.011-0.075; Table 2). The highest value (0.119) was obtained from threshold animal model A. Important differences were found among model specifications. In all cases, models A resulted in the highest heritability and models $\mathrm{D}$ in the lowest. The addition of the permanent environmental effect of the cow to the analysis (model B) significantly decreased the heritability of CS estimated using animal models. The inclusion of maternal genetic effect (model C) did not significantly affect heritability of CS compared to model B, even though a small decrease was observed in the threshold animal model. Finally, the addition of the permanent environmental effect of the service sire led to a notable erosion of the direct genetic variance, leading to much lower heritability compared to all previous models.

The posterior means of heritability for NR56 (Table 3) resembled those described for CS. Threshold gave higher heritabilities than linear models $(0.010-0.054$ vs. 0.005-0.041), and animal resulted in higher values than sire models (0.014-0.054 vs. 0.005-0.032). Again, the highest heritability (0.054) was obtained from threshold animal model A. Models A resulted in the highest heritabilities, followed by models B and C, which produced very similar results. Heritability significantly decreased in models D, as a consequence of the inclusion of the permanent environmental effect of service sire, which eroded a large portion of direct genetic variance.
Our heritability estimates appear to fall between the extremely low values reported by VanRaden and Miller (2006) for embryo and fetal loss in US Holsteins, and the higher (although still moderate) values found by Bamber et al. (2009) for pregnancy loss. If EES is compared to bull fertility as a trait of the potential calf, Jansen (1986), in a study on Dutch Friesian AI bulls, estimated heritabilities between 0.015 and 0.024 for CS, and $0.013-0.021$ for NR56 using linear sire models including direct and maternal effects. The results for NR56 is comparable to our finding $(0.020$; Table 3) obtained using model A, whereas heritability for CS is much lower than our estimate (0.045; Table 2). For CS, both Kuhn and Hutchinson (2008) and Berry et al. (2011) reported heritabilities below 0.010 , i.e., much lower than ours, although these studies are not directly comparable for to the different methodologies involved. Nadarajah et al. (1988) estimated heritability of 0.150 , but via sire-son regression. For NR56, Andersen-Ranberg et al. (2003) found values around 0.010 for direct heritability on heifers, whereas our comparable value was 0.020 (Table 3), although we used inseminations both on heifers and cows.

Regarding the comparison between linear and threshold models, our findings are in accordance to Andersen-Ranberg et al. (2005), Weller and Ron (1992), Matos et al. (1997b), and Weigel and Rekaya (2000), who found higher heritability from threshold models for female fertility. It is well known that the use of linear models with categorical data ignores their non-continuous distribution and tends to underestimate heritability (Gianola, 1982).

Matos et al. (1997b), working on calving rate of Rambouillet and Finnsheep sheep, estimated higher heritabilities from animal than sire models, and from threshold than linear models, similar to the pattern found in our study, although sheep fertility from artificial insemination is not directly comparable. 
Table 3

Estimates $^{\mathrm{a}}$ of variance components and heritability for non-return at $56 \mathrm{~d}$ after service (NR56) from different models ${ }^{\mathrm{c}}$.

\begin{tabular}{|c|c|c|c|c|c|c|c|c|c|c|}
\hline \multicolumn{9}{|c|}{ (Co)variance components ${ }^{\mathrm{b}}$} & \multicolumn{2}{|c|}{ Heritability } \\
\hline & $\sigma_{d g}^{2}$ & $\sigma_{c o}^{2}$ & $\sigma^{2}{ }_{m g}$ & $\sigma_{d g-m g}$ & $\sigma_{s s}^{2}$ & $\sigma^{2} h e$ & $\sigma^{2}{ }_{t c}$ & $\sigma^{2}{ }_{e}$ & $h^{2}$ & HPD95 \\
\hline \multicolumn{11}{|c|}{ Linear sire model } \\
\hline A & 0.0011 & & & & & 0.0041 & 0.0008 & 0.2045 & 0.020 & $0.013 ; 0.028$ \\
\hline B & 0.0010 & 0.0054 & & & & 0.0036 & 0.0008 & 0.1994 & 0.020 & $0.013 ; 0.028$ \\
\hline $\mathrm{C}$ & 0.0010 & 0.0050 & 0.0005 & 0.0000 & & 0.0036 & 0.0008 & 0.1994 & 0.020 & $0.013 ; 0.028$ \\
\hline $\mathrm{D}$ & 0.0003 & 0.0050 & 0.0005 & 0.0000 & 0.0006 & 0.0036 & 0.0008 & 0.1994 & 0.005 & $0.001 ; 0.013$ \\
\hline \multicolumn{11}{|c|}{ Linear animal model } \\
\hline A & 0.0086 & & & & & 0.0038 & 0.0008 & 0.1984 & 0.041 & $0.031 ; 0.050$ \\
\hline B & 0.0043 & 0.0042 & & & & 0.0036 & 0.0008 & 0.1976 & 0.021 & $0.015 ; 0.027$ \\
\hline $\mathrm{C}$ & 0.0046 & 0.0030 & 0.0036 & -0.0023 & & 0.0035 & 0.0008 & 0.1973 & 0.022 & $0.015 ; 0.028$ \\
\hline D & 0.0030 & 0.0032 & 0.0029 & -0.0012 & 0.0004 & 0.0035 & 0.0008 & 0.1980 & 0.014 & $0.008 ; 0.022$ \\
\hline \multicolumn{11}{|c|}{ Threshold sire model } \\
\hline A & 0.0085 & & & & & 0.0361 & 0.0066 & 1 & 0.032 & $0.021 ; 0.045$ \\
\hline B & 0.0085 & 0.0438 & & & & 0.0335 & 0.0068 & 1 & 0.031 & $0.020 ; 0.044$ \\
\hline $\mathrm{C}$ & 0.0086 & 0.0402 & 0.0045 & -0.0003 & & 0.0333 & 0.0068 & 1 & 0.032 & $0.021 ; 0.044$ \\
\hline $\mathrm{D}$ & 0.0026 & 0.0401 & 0.0044 & -0.0001 & 0.0052 & 0.0332 & 0.0068 & 1 & 0.010 & $0.002 ; 0.024$ \\
\hline \multicolumn{11}{|c|}{ Threshold animal model } \\
\hline A & 0.0595 & & & & & 0.0349 & 0.0070 & 1 & 0.054 & $0.043 ; 0.066$ \\
\hline B & 0.0325 & 0.0349 & & & & 0.0332 & 0.0070 & 1 & 0.029 & $0.022 ; 0.038$ \\
\hline$C$ & 0.0327 & 0.0249 & 0.0273 & -0.0147 & & 0.0325 & 0.0069 & 1 & 0.029 & $0.021 ; 0.037$ \\
\hline $\mathrm{D}$ & 0.0198 & 0.0248 & 0.0258 & -0.0094 & 0.0039 & 0.0325 & 0.0690 & 1 & 0.018 & $0.001 ; 0.026$ \\
\hline
\end{tabular}

a Estimates are the means of the marginal posterior densities for variance components and the mean for the heritability; HPD95: lower and upper bound of the 95\% highest posterior density region.

${ }^{\mathrm{b}} \sigma_{d g}^{2}$ is the direct genetic variance; $\sigma_{c o}^{2}$ is the permanent environmental variance of the cow; $\sigma^{2}{ }_{m g}$ is the maternal genetic variance; $\sigma_{d g-m g}$ is the direct-maternal genetic covariance; $\sigma_{s s}^{2}$ is the permanent environmental variance of the service sire; $\sigma^{2}{ }_{h e}$ is the herd variance; $\sigma^{2}$ ic is the technician variance; $\sigma^{2}{ }_{e}$ is the residual variance.

c All models account for the 'fixed' effects of year-season of insemination, parity by days in milk of cow at insemination (age at insemination for heifers), status of the

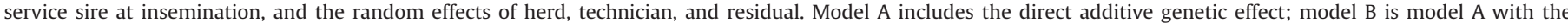

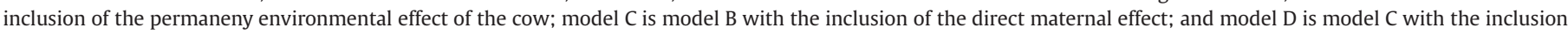
of the permanent environmental effect of the service sire.

\subsection{Goodness of fit and predictive ability}

\subsubsection{Goodness of fit}

In the present study, the MSE from local weighted regression was used as indicator of goodness of fit for CS (Table 4) and NR56 (Table 5). Values of MSE were smaller for NR56 than CS, but the former showed also a lower SD (0.46) than the latter (0.50). Within trait, no significant differences in goodness of fit were reported between linear and threshold models, whereas sire models resulted in significantly lower values of MSE than animal models. Overall, models D+ fit better than others, whereas models D fit worse than A-C; this is mainly because models A-C included part of the permanent environmental variance of service sire into the additive genetic one. For both fertility traits, linear sire model D+ gave the best fitting ( 0.287 for CS and 0.179 for NR56), followed by linear sire model B (0.389 for CS and 0.201 for NR56).

\subsubsection{Predictive ability}

Sum of $\chi^{2}$ and WP were used as indicators of predictive ability of models for CS (Table 4) and NR56 (Table 5). The prediction of male fertility for young bulls was obtained for 72 service sires and was based on the performance of their ancestors, whereas the prediction for male fertility of proven bulls was obtained on 38 service sires having at least 100 observations in DATA $_{05}$ and 37 observations in DATA $\mathrm{D}_{08}$, and was based on their own performance from 1999 to 2005. Regardless of the status of the service sire (young or proven), CS was much more predictable than NR56, in agreement with the heritability estimates, which were higher for the former (Table 2) than for the latter (Table 3) fertility trait.

Concerning young bulls, values of sum of $\chi^{2}$ and WP for NR56 were almost flat across the models (Table 5), whereas for CS slightly better predictions were generally obtained for animal models than sire models (Table 4). Overall, linear models performed similarly to threshold models, suggesting that they were robust in predicting male fertility. No recognizable patterns were highlighted among model specifications. It should be noted that models D + were not considered for young bulls, as no prediction of the permanent environmental effect of the service sire was available. According to the sum of $\chi^{2}$, the best predictive ability for CS was achieved using the threshold animal model B, whereas on the basis of WP, the threshold animal model D performed best. For NR56, linear animal model B performed better than the others, both in terms of sum of $\chi^{2}$ and WP. However, the differences among models in predictive ability for NR56 were quite low.

In the case of proven bulls, no well-defined patterns were identified among models within trait. According to the sum of $\chi^{2}$, the best predictive ability for CS and NR56 was reached through threshold animal model D and threshold sire model D, respectively (Tables 4 and 5), whereas in terms of WP, the best performance for CS and NR56 were obtained through linear sire model D and threshold sire model $\mathrm{D}$, respectively (Tables 4 and 5). To our knowledge, no comparable assessment of predictive ability for bull fertility is available in literature. Nonetheless, Kuhn and Hutchinson (2008) reported correlations between predicted and observed bull fertility between 0.283 and 0.381 . 
Table 4

Summary of statistics for the cross-validation applied to calving per service. In bold the values referring to the best model $^{\mathrm{a}}$.

\begin{tabular}{|c|c|c|c|c|c|}
\hline \multirow[t]{2}{*}{ Model $^{\mathrm{b}}$} & \multirow{2}{*}{$\begin{array}{l}\text { Goodness of } \\
\mathrm{fit}^{\mathrm{C}} \\
\text { MSE }\end{array}$} & \multicolumn{2}{|c|}{$\begin{array}{l}\text { Prediction }{ }^{\mathrm{d}} \text { of young } \\
\text { bulls }^{\mathrm{e}}\end{array}$} & \multicolumn{2}{|c|}{$\begin{array}{l}\text { Prediction }{ }^{\mathrm{d}} \text { of proven } \\
\text { bulls }^{\mathrm{f}}\end{array}$} \\
\hline & & Sum of $\chi^{2}$ & WP & Sum of $\chi^{2}$ & WP \\
\hline \multicolumn{6}{|c|}{ Linear sire model } \\
\hline A & 0.396 & 217,362 & 0.138 & $1,412,860$ & 0.128 \\
\hline B & 0.389 & 175,458 & 0.128 & $1,112,976$ & 0.114 \\
\hline $\mathrm{C}$ & 0.398 & 222,260 & 0.140 & $1,467,384$ & 0.130 \\
\hline $\mathrm{D}$ & 0.658 & 167,393 & 0.125 & $1,066,642$ & 0.101 \\
\hline $\mathrm{D}+$ & 0.287 & & & $1,302,014$ & 0.120 \\
\hline \multicolumn{6}{|c|}{ Linear animal model } \\
\hline A & 0.438 & 164,757 & 0.126 & $1,203,426$ & 0.121 \\
\hline B & 0.530 & 178,683 & 0.128 & $1,315,672$ & 0.117 \\
\hline $\mathrm{C}$ & 0.617 & 166,429 & 0.123 & $1,323,049$ & 0.118 \\
\hline $\mathrm{D}$ & 0.811 & 168,465 & 0.125 & $1,298,009$ & 0.122 \\
\hline $\mathrm{D}+$ & 0.405 & & & $1,431,006$ & 0.127 \\
\hline \multicolumn{6}{|c|}{ Threshold sire model } \\
\hline A & 0.394 & 193,809 & 0.133 & $1,439,904$ & 0.130 \\
\hline B & 0.428 & 171,107 & 0.127 & $1,147,294$ & 0.115 \\
\hline $\mathrm{C}$ & 0.417 & 195,214 & 0.135 & $1,441,360$ & 0.127 \\
\hline $\mathrm{D}$ & 0.634 & 160,361 & 0.124 & $1,903,617$ & 0.135 \\
\hline $\mathrm{D}+$ & 0.335 & & & $1,465,459$ & 0.127 \\
\hline \multicolumn{6}{|c|}{ Threshold animal model } \\
\hline A & 0.458 & 173,207 & 0.128 & $1,295,535$ & 0.120 \\
\hline $\mathrm{B}$ & 0.483 & 159,219 & 0.125 & $1,252,269$ & 0.116 \\
\hline C & 0.624 & 170,647 & 0.126 & $1,270,588$ & 0.115 \\
\hline $\mathrm{D}$ & 0.931 & 167,726 & 0.122 & $1,064,837$ & 0.110 \\
\hline $\mathrm{D}+$ & 0.468 & & & $1,476,624$ & 0.128 \\
\hline
\end{tabular}

${ }^{a}$ If $\mathrm{D}+$ showed the best values, the best model excluding $\mathrm{D}+$ is highlighted as well.

${ }^{b}$ All models account for the 'fixed' effects of year-season of insemination, parity by days in milk of cow at insemination (age at insemination for heifers), status of the service sire at insemination, and the random effects of herd, technician, and residual. Model A includes the direct additive genetic effect; model B is model A with the inclusion of the permanent environmental effect of the cow; model C is model B with the inclusion of the direct maternal effect; and model D is model $C$ with the inclusion of the permanent environmental effect of the service sire. In all models the predictor is the service sire EBV from the direct additive genetic effect. In $\mathrm{D}+$, the predictor is the sum of the EBV and the solution of each service sire for the permanent environmental effect of the service sire.

${ }^{c}$ Mean squared error estimated via non-parametric local weighted regression of raw calving per service on EBV of the sire for each model.

${ }^{\mathrm{d}}$ Predictive ability is measured according to: sum of $\gamma 2$ statistics across service sires, based on the comparison between the predicted and observed outcome of the insemination for each service sire; and percentage of wrong predictions on total predictions (WP), as the number of wrongly predicted successful inseminations on total number of inseminations.

e Based on service sires EBV obtained via relationship matrix in calibration dataset and validated accordingly to their inseminations in validation dataset $(n=72)$.

${ }^{\mathrm{f}}$ Based on service sires EBV obtained on a minimum of 100 inseminations in calibration dataset and 30 inseminations in validation dataset $(n=37)$.

\section{Conclusions}

In the present study, (co)variance components and predictive ability of embryo survival as a trait for the potential calf from several models were obtained. Genetic variance for this trait exist in the Italian Brown Swiss population. In general, estimates of heritability for CS and NR56 were higher when calculated using (co)variance components from animal models, particularly from threshold models. Even if NR56 is available earlier and requires only the registration of inseminations, CS is characterized by higher heritability and lower incidence of wrong predictions, thus it seems the preferred trait to be chosen for selection purposes.
Table 5

Summary of statistics for the cross-validation applied to non-return at $56 \mathrm{~d}$ after service. In bold the values referring to the best model ${ }^{\mathrm{a}}$.

\begin{tabular}{|c|c|c|c|c|c|}
\hline \multirow[t]{2}{*}{ Model $^{\mathrm{b}}$} & \multirow{2}{*}{$\begin{array}{l}\text { Goodness of } \\
\text { fit }^{\mathrm{c}} \\
\text { MSE }\end{array}$} & \multicolumn{2}{|c|}{$\begin{array}{l}\text { Prediction }{ }^{\mathrm{d}} \text { of young } \\
\text { bulls }^{\mathrm{e}}\end{array}$} & \multicolumn{2}{|c|}{$\begin{array}{l}\text { Prediction }{ }^{\mathrm{d}} \text { of proven } \\
\text { bulls }^{\mathrm{f}}\end{array}$} \\
\hline & & Sum of $\chi^{2}$ & WP & Sum of $\chi^{2}$ & WP \\
\hline \multicolumn{6}{|c|}{ Linear sire model } \\
\hline A & 0.209 & $1,977,398$ & 0.384 & $12,985,864$ & 0.378 \\
\hline B & 0.201 & $1,943,405$ & 0.381 & $13,499,231$ & 0.383 \\
\hline $\mathrm{C}$ & 0.205 & $1,962,790$ & 0.383 & $12,990,475$ & 0.374 \\
\hline $\mathrm{D}$ & 0.351 & $1,946,272$ & 0.382 & $14,070,224$ & 0.397 \\
\hline $\mathrm{D}+$ & 0.179 & & & $13,259,839$ & 0.379 \\
\hline
\end{tabular}

\begin{tabular}{lrrrrr}
\multicolumn{2}{l}{ Linear } & animal model & & & \\
A & 0.302 & $1,985,109$ & 0.385 & $13,378,121$ & 0.379 \\
B & 0.480 & $\mathbf{1 , 9 3 1 , 2 2 8}$ & $\mathbf{0 . 3 8 0}$ & $13,319,827$ & 0.379 \\
C & 0.486 & $1,966,864$ & 0.383 & $13,698,249$ & 0.386 \\
D & 0.516 & $1,948,416$ & 0.381 & $13,141,642$ & 0.380 \\
D & 0.337 & & & $12,968,669$ & 0.376
\end{tabular}

\begin{tabular}{|c|c|c|c|c|c|}
\hline \multicolumn{6}{|c|}{ Threshold sire model } \\
\hline A & 0.214 & $1,951,937$ & 0.383 & $12,887,053$ & 0.378 \\
\hline B & 0.222 & $1,984,740$ & 0.387 & $13,503,749$ & 0.383 \\
\hline C & 0.245 & $1,959,796$ & 0.383 & $13,046,785$ & 0.377 \\
\hline D & 0.372 & $2,062,731$ & 0.390 & $12,141,282$ & 0.366 \\
\hline $\mathrm{D}+$ & 0.184 & & & $12,930,594$ & 0.377 \\
\hline
\end{tabular}

\begin{tabular}{llllll}
\multicolumn{2}{l}{ Threshold animal model } & & & & \\
$\mathrm{A}$ & 0.370 & $1,945,611$ & 0.382 & $13,437,804$ & 0.386 \\
$\mathrm{~B}$ & 0.468 & $1,983,425$ & 0.384 & $13,455,974$ & 0.382 \\
$\mathrm{C}$ & 0.468 & $1,939,165$ & 0.383 & $13,056,420$ & 0.376 \\
$\mathrm{D}$ & 0.597 & $1,958,873$ & 0.386 & $14,105,815$ & 0.388 \\
$\mathrm{D}+$ & 0.359 & & & $13,430,642$ & 0.383 \\
\hline
\end{tabular}

${ }^{\text {a }}$ If $\mathrm{D}+$ showed the best values, the best model excluding $\mathrm{D}+$ is highlighted as well.

b All models account for the 'fixed' effects of year-season of insemination, parity by days in milk of cow at insemination (age at insemination for heifers), status of the service sire at insemination, and the random effects of herd, technician, and residual. Model A includes the direct additive genetic effect; model B is model A with the inclusion of the permanent environmental effect of the cow; model C is model B with the inclusion of the direct maternal effect; and model D is model $C$ with the inclusion of the permanent environmental effect of the service sire. In all models the predictor is the service sire EBV from the direct additive genetic effect. In $\mathrm{D}+$, the predictor is the sum of the EBV and the solution of each service sire for the permanent environmental effect of the service sire.

${ }^{c}$ Mean squared error estimated via non-parametric local weighted regression of raw non-return at $56 \mathrm{~d}$ after service on EBV of the sire for each model.

${ }^{\mathrm{d}}$ Predictive ability is measured according to: sum of $\gamma 2$ statistics across service sires, based on the comparison between the predicted and observed outcome of the insemination for each service sire; and percentage of wrong predictions on total predictions (WP), as the number of wrongly predicted successful inseminations on total number of inseminations.

e Based on service sires EBV obtained via relationship matrix in calibration dataset and validated accordingly to their inseminations in validation dataset $(n=72)$.

${ }^{\mathrm{f}}$ Based on service sires EBV obtained on a minimum of 100 inseminations in calibration dataset and 30 inseminations in validation dataset $(n=37)$.

Predictive ability did not differ significantly among approaches, thus suggesting that the use of the most parsimonious approaches might be advised to reduce computational time while providing similar reliability of prediction.

\section{Conflict of interests}

The authors confirm that there is no conflict of interest associated with the publication of this manuscript. Funding sources had no involvement in study design, in collection, analysis and interpretation of data and in writing of the report. 


\section{Acknowledgments}

Authors thank the Trento Province (Italy) for financial support, the Breeders Association of Bolzano-Bozen province (Italy) for providing field data, the Italian Brown Swiss Cattle Breeders Association (ANARB, Verona, Italy) for supplying pedigree information, and the Superbrown Consortium of Bolzano and Trento (Italy) for technical support.

\section{References}

Andersen-Ranberg, I.M., Heringstad, B., Gianola, D., Chang, Y.M., Klemetdal, G., 2005. Comparison between bivariate models for 56-d nonreturn and interval from calving to first insemination in Norwegian Red. J. Dairy Sci. 88, 2190-2198.

Andersen-Ranberg, I.M., Heringstad, B., Klemetsdal, G., Svendsen, M., Steine, T., 2003. Heifer fertility in Norwegian dairy cattle: variance components and genetic change. J. Dairy Sci. 86, 2706-2714.

Ayalon, N., 1978. A review of embryonic mortality in cattle. J. Reprod. Fertil. 54 (2), 483-493.

Azzam, S.M., Keele, J.W., Nielsen, M.K., 1988. Expectations of heritability estimates for non-return rate of bulls and conception rate of cows. J. Anim. Sci. 66, 2767-2783.

Bamber, R.L., Shook, G.E., Wiltbank, M.C., Santos, J.E.P., Fricke, P.M., 2009. Genetic parameters for anovulation and pregnancy loss in dairy cattle. J. Dairy Sci. 92 (11), 5739-5753.

Berry, D.P., Evans, R.D., Mc Parland, S., 2011. Evaluation of bull fertility in dairy and beef cattle using cow field data. Theriogenology 75, 172-181.

Blaschek, M., Kaya, A., Zwald, N., Memili, E., Kirkpatrick, B.W., 2011. A whole-genome association analysis of noncompensatory fertility in Holstein bulls. J. Dairy Sci. 94, 4695-4699.

Caraviello, D.Z., Weigel, K.A., Gianola, D., 2004. Comparison between a Weibull proportional hazards model and a linear model for predicting the genetic merit of US Jersey sires for daughter longevity. J. Dairy Sci. 87, 1469-1476.

Cecchinato, A., González-Recio, O., López de Maturana, E., Gallo, L., Carnier, P., 2010. A comparison between different survival and threshold models with an application to piglet preweaning survival in a dry-cured ham-producing crossbred line. J. Anim. Sci. 88, 1990-1998.

Clay, J.S., McDaniel, B.T., 2001. Computing mating bull fertility from DHI nonreturn data. J. Dairy Sci. 84, 1238-1245.

Cleveland, W.S., Loader, C.L., 1996. Smoothing by local regression. Principles and methods. In: Haerdle, W., Schimek, M.G. (Eds.), Statistical Theory and Computational Aspects of Smoothing. Springer, New York, NY, pp. 10-49.

Dal Zotto, R., Penasa, M., De Marchi, M., Cassandro, M., López-Villalobos, N., Bittante, G., 2009. Use of crossbreeding with beef bulls in dairy herds: Effect on age, body weight, price, and market value of calves sold at livestock auctions. J. Anim. Sci. 87, 3053-3059.

Geweke, J., 1992. Evaluating the accuracy of sampling-based approaches to the calculation of posterior moments (with discussion). In: Berger, J.O., Bernardo, J. M., Dawid, A.P., Smith, A.F.M. (Eds.), Bayesian Statistics. Oxford University Press, Oxford, UK, pp. 164-193.

Geyer, C.J., 1992. Practical Markov chain Monte Carlo. Stat. Sci. 7, 473-483.

Gianola, D., 1982. Theory and analysis of threshold characters. J. Anim. Sci. 54, 1079-1096.
González-Recio, O., Chang, Y.M., Gianola, D., Weigel, K.A., 2005. Number of inseminations to conception in Holstein cows using censored records and timedependent covariates. J. Dairy Sci. 88, 3655-3662.

Hyppänen, K., Juga, J., 1998. Environmental and genetic effects on the 60-d nonreturn rate in Finnish AI bulls. Interbull Bull 18, 91-98.

Jansen, J., 1986. Direct and maternal genetic parameters of fertility traits in Friesian cattle. Livest. Prod. Sci. 15, 153-164.

Kuhn, M.T., Hutchinson, J.L., 2008. Prediction of dairy bull fertility from field data: use of multiple services and identification and utilization of factors affecting bull fertility. J. Dairy Sci. 91, 2481-2492.

Legarra, A., Varona, L., Lopez de Maturana, E., 2008. TM Threshold Model. 〈http:// snp.toulouse.inra.fr/ alegarra/manualtm.pdf (accessed 26.10.10).

López-Gatius, F., Santolaria, P., Yaniz, J., Rutllant, J., López-Béjar, M., 2002. Factors affecting pregnancy loss from gestation days 38 to 90 in lactating dairy cows from a single herd. Theriogenology 57 (4), 1251-1261.

Lucy, M.C., 2001. Reproductive loss in high-producing dairy cattle: where will it end. J. Dairy Sci. 84, 1277-1293.

Matos, C.A.P., Thomas, D.L., Gianola, D., Perez-Enciso, M., Young, L.D., 1997a. Genetic analysis of discrete reproductive traits in sheep using linear and nonlinea models: II. Goodness of fit and predictive ability. J. Anim. Sci. 75, 88-94.

Matos, C.A.P., Thomas, D.L., Gianola, D., Tempelman, R.J., Young, L.D., 1997b. Genetic analysis of discrete reproductive traits in sheep using linear and nonlinear models: I. Estimation of genetic parameters. J. Anim. Sci. 75, 76-87.

Nadarajah, K., Burnside, E.B., Schaeffer, L.R., 1988. Genetic parameters for fertility of dairy bulls. J. Dairy Sci. 71, 2730-2734.

Norman, H.D., Wright, J.R., Kuhn, M.T., Hubbard, S.M., Cole, J.B., VanRaden, P.M., 2009. Genetic and environmental factors that impact gestation length in dairy cattle. J. Dairy Sci. 92, 2259-2269.

Penasa, M., De Marchi, M., Dal Zotto, R., Cecchinato, A., Cassandro, M., Bittante, G. 2009. Influence of the sire on market value of Belgian Blue x Brown Swiss crossbred calves. Ital. J. Anim. Sci 8 (Suppl. 3), S113-S115.

Pryce, J.E., Royal, M.D., Garnsworthy, P.C., Mao, I.L., 2004. Fertility in the high producing dairy cow. Livest. Prod. Sci. 86, 125-135.

Royal, M.D., Darwash, A.O., Flint, A.P.F., Webb, R., Woolliams, J.A., Lamming, G.E., 2000. Declining fertility in dairy cattle: changes in traditional and endocrine parameters of fertility. Anim. Sci. 70 (487-501).

Starbuck, M.J., Dailey, R.A., Inskeep, E.K., 2004. Factors affecting retention of early pregnancy in dairy cattle. Anim. Reprod. Sci. 84 (1), 27-39.

Sun, C., Su., G., 2010. Comparison on models for genetic evaluation of non-return rate and success in first insemination of the Danish Holstein cows. Livest. Sci. $127,205-210$

Tiezzi, F., Maltecca, C., Penasa, M., Cecchinato, A., Bittante, G., 2013. Short communication: Genetic analysis of dairy bull fertility from field data of Brown Swiss cattle. J. Dairy Sci. 96, 7325-7328.

VanRaden, P.M., Miller, R.H., 2006. Effects of nonadditive genetic interactions, inbreeding, and recessive defects on embryo and fetal loss by seventy days. J. Dairy Sci. 89, 2716-2721.

Vazquez, A.I., Perez-Cabal, M.A., Heringstad, B., Rodrigues-Motta, M., Rosa, G.J.M. Gianola, D., Weigel, K.A., 2012. Predictive ability of alternative models for genetic analysis of clinical mastitis. J. Anim. Breed. Genet. 129, 120-128.

Veerkamp, R.F, Beerda, B, van der Lende, T, 2003. Effects of genetic selection for milk yield on energy balance, levels of hormones, and metabolites in lactating cattle, and possible links to reduced fertility. Livest. Prod. Sci. 83, 257-275.

Weigel, K.A., Rekaya, R., 2000. Genetic parameters for reproductive traits of Holstein cattle in California and Minnesota. J. Dairy Sci. 83, 1072-1080.

Weller, J.I., Ron, M., 1992. Genetic analysis of fertility traits in Israeli Holsteins by linear and threshold models. J. Dairy Sci. 75, 2541-2548. 